\title{
Development of a screening and recruitment registry to facilitate perinatal depression research in obstetrics settings in the USA
}

\author{
Lucy J. Allbaugh $^{\text {a,* }}$, Sheila M. Marcus ${ }^{\text {b }}$, Eleanor C. Ford ${ }^{c}$, Heather A. Flynn ${ }^{\text {b,d }}$ \\ a Department of Psychology, Miami University, Oxford, OH, USA \\ b Department of Psychiatry, University of Michigan Medical School, Ann Arbor, MI, USA \\ c Department of Psychology, Teachers College at Columbia University, New York, NY, USA \\ d Department of Behavioral Sciences and Social Medicine, The Florida State University College of Medicine, Tallahassee, FL, USA
}

\section{A R T I C L E I N F O}

\section{Article history:}

Received 23 May 2014

Received in revised form 2 September 2014

Accepted 28 October 2014

\section{Keywords:}

Obstetrics

Perinatal depression

Research recruitment

Screening

\begin{abstract}
A B S T R A C T
Objective: To create a multi-site registry to enable future large-scale studies of perinatal depression among women attending obstetrics clinics in the USA. Methods: A screening and recruitment registry was developed that included women aged at least 18 years who attended seven obstetric clinics in the University of Michigan Health System (Ann Arbor, MI, USA) for prenatal care between September 8, 2008, and June 9, 2011. Participants completed depression screening and research recruitment materials. Results: Of 4745 women who returned a screening form, 2983 had completed it, giving an overall agreement rate of $62.9 \%$. A total of 630 participants were enrolled into ten research studies via the registry. Among the 2982 women for whom scores on the Edinburgh Postnatal Depression Scale were available, 494 (16.6\%) fell within the at-risk range or had scores suggestive of clinical depression. Conclusion: The present registry could improve detection of perinatal depression symptoms and potentially serve as a model for dissemination and implementation at other sites with an interest in studying factors linked to perinatal depression.

(C) 2014 International Federation of Gynecology and Obstetrics. Published by Elsevier Ireland Ltd. All rights reserved.
\end{abstract}

\section{Introduction}

Worldwide, major depressive disorder is one of the main causes of disability-adjusted life years (years of healthy life lost), with women disproportionately affected $[1,2]$. The peak prevalence for depressive disorders among women occurs during the childbearing years [3,4], with the recorded prevalence as high as $22.2 \%$ in pregnancy and the postpartum period $[5,6]$.

Depression during pregnancy-which has been variously defined-has been linked to negative birth outcomes [7] and effects on the infant's temperament [8]. Prenatal care settings provide an ideal opportunity for intervention, but most women who present with symptoms of depression at such centers in the USA do not receive adequate treatment. One study evaluating the ability of US obstetrics clinics to detect symptoms of depression [9] found that just $14 \%$ of women with an elevated score on the Edinburgh Postnatal Depression Scale (EPDS) [10] were receiving any kind of mental health care, such as antidepressant medications or psychotherapy.

Therefore, screening for depression has been widely supported as part of routine obstetric care [11]. Nevertheless, recommendations published in 2010 by the American College of Obstetricians and

\footnotetext{
* Corresponding author at: Department of Psychology, Miami University, 90 North Patterson Avenue, Oxford, OH 45056, USA. Tel.: + 1734417 3073; fax: + 15135292420.

E-mail address: allbaulj@miamioh.edu (L.J. Allbaugh).
}

Gynecologists [12] cautioned that screening alone is insufficient to address perinatal depression and that this approach offers potential benefit only when closely linked with appropriate intervention. Systematic screening is not routinely associated with adequate follow-up and improved clinical outcomes in the USA $[13,14]$. Furthermore, intervention research can be limited by difficulties in recruitment. This difficulty might be related to a lack of coordinated recruitment efforts and/or to the length of time required to identify a large number of participants who meet study criteria [15].

Some studies have reported on rates of agreement to participate in intervention trials among women already screened for perinatal depression [16,17], but few have recorded rates of agreement to screening procedures themselves among women attending obstetrics clinics who are approached to participate in research [18-20]. Consequently, the findings of research studies that target pregnant women who are experiencing depression (particularly untreated major depressive disorder) are often limited by small or homogeneous sample populations [21,22].

The development of screening procedures that are both feasible within clinic settings and efficient as research recruitment tools is an important step toward improved screening, detection, and intervention research. Although many investigators already use screening tools for research recruitment in obstetrics settings, standardized, multicenter screening efforts are urgently needed to enable large-scale recruitment while minimizing the burden on clinical staff and their patients. Australia, Canada, the UK, and several Scandinavian countries benefit 
from the availability of national healthcare or population-based registries that make the process of conducting research with large, representative cohorts easily accessible $[23,24]$. No such standard registries currently exist in the USA; guidance on implementation and acceptability of such procedures could help to accelerate the pace of US-based research. Importantly, assessing large and diverse samples of patients attending obstetrics services could allow researchers to characterize phenotypes of perinatal depression outside of specialty psychiatric care.

The present study aimed to develop a screening and recruitment registry of women interested in research participation to be used in obstetric clinics at multiple centers in the USA. The first goal was to provide instruction and guidance on optimizing screening and recruitment registry procedures across multiple settings. The second goal was to provide data on how the screening process could enhance recruitment to multiple research studies by streamlining the process to minimize burden on clinic staff and patients.

\section{Materials and methods}

A screening and recruitment registry was developed using data provided by women who attended one of seven obstetrics clinics within the University of Michigan Health System (Ann Arbor, MI, USA) between September 8, 2008, and June 9, 2011. All procedures were approved by the institutional review board of the University of Michigan Medical School.

The present study commenced with consultations with obstetrics clinic stakeholders regarding the feasibility of developing a registry. Full support for the project was crucial at all stages to ensure that the registry could be embedded within existing systems (e.g. in other healthcare screening or during regular prenatal care visits). The concerns of clinic staff mainly related to the potential time required to administer screening, return forms to research staff, and enter additional information (e.g. EPDS score) into medical records. Therefore, research staff offered a menu of procedures to each clinic from which to customize registry operations (e.g. options on how to administer EPDS). Connection with clinical trials was an integral part of the registry for those agreeing to participate. Additionally, clinics were aided in referring women to existing mental health services. Staff members at participating clinics were instructed on EPDS scoring and interpretation. Clinics were provided with a comprehensive list of mental health services available to women found to have significant depressive symptoms.

After consultation, consent forms (to establish willingness to be included in the registry) and screening forms (to gather information about depressive symptoms and other eligibility criteria) were developed and iteratively updated. Pregnant women aged at least 18 years who presented for prenatal care at one of the participating obstetrics clinics were eligible for inclusion in the registry. The consent and screening forms were attached to the EPDS test administered by clinic staff. The women were provided with a brief description of the registry and informed about the need for ongoing research; however, in view of the changing nature of research within the psychiatry department, a description of the specific studies recruiting was not provided. They were also advised that should they be deemed eligible to participate in a specific study, they would be contacted by a member of the relevant research team, that they could decide at that time whether they would like to participate in a trial, and that no cost would be associated with such participation. Three options were provided for level of participation in the registry: (1) complete both the consent and screening forms to enter the registry and potentially be contacted for participation in specific research projects (participants); (2) check an "opt out" box on the consent form to provide anonymous data to the registry only at the time of screening (anonymous participants); or (c) decline participation by leaving the consent form blank (refusers).

Version 1 of the screening form was concise and did not require a separate consent form because it was intended solely to recruit for an existing project [25] within the University of Michigan Health System, which had its own consent process. Version 2 included a consent form and was also longer than version 1 to accommodate inclusion criteria for additional studies. The final screening form (version 3 ) was the shortest because it streamlined the consent and screening forms and removed items that could easily be found in medical records (Supplementary Material S1). The consent process allowed for examination of the medical records to determine eligibility for specific studies. Two versions of the screening and recruitment system indicated that psychoeducational materials about perinatal depression could be provided to women by the research team upon request, and this offer was made irrespective of participation in the registry.

As noted, three versions of the registry screening form were used to gather information not found in the medical record. Lifetime depression and episodes of depression within the past 6 months were assessed using depression-focused items derived from the Diagnostic Interview Schedule, Version Three, Revised [26]. The sensitivity of these items to screen for depression was found to range between 0.83 and 0.94 in a community sample of adults in three major cities in the USA [27]. Current symptoms of depression were assessed by the EPDS [10]. This tool is a widely used, reliable, and validated 10-item measure of perinatal mood symptoms; the EPDS was approved by the obstetrics clinics participating in the present study as the standard screening tool to be used for the registry. Total EPDS scores range from 0 to 30 , with high scores indicating increased symptoms of depression [10]. A score at or above nine indicates a risk of major depressive disorder, whereas a score at or above 12 indicates that the diagnostic criteria have probably been met [10].

The EPDS was scored, reviewed, and entered into the medical record by a nurse, medical assistant, or obstetrician who addressed the EPDS results, including indicated risk (e.g. suicide), and provided standard care, including referral to mental health resources. EPDS and screening forms were returned to the research staff and data entered into an SPSS (version 20; IBM, Armonk, NY, USA) tracking database by the registry coordinator who determined eligibility for participation in ongoing clinical research programs; eligible participants were referred to the relevant investigators for enrollment. The prevalence of depression symptoms among the registry population was also evaluated as part of the present study.

Data were analyzed using SPSS version 20 (IBM, Armonk, NY, USA). Agreement rates were determined by dividing the number of women who completed the forms by the number eligible to undergo screening. Frequency data were calculated to describe eligibility for, and participation in, individual research studies. The mean total EPDS score, frequency of endorsement of individual EPDS items, history of depression, and current use of psychotherapy or psychopharmacological treatment were calculated.

\section{Results}

A total of 4745 screening forms were collected during the present study period. The mean age at the time of screening was $30.3 \pm 5.0$ years (range 18.0-46.0) and mean gestational age was $24.2 \pm 9.1$ weeks (range 0.0-40.0)

Of the women screened, 2259 (47.6\%) provided screening data and agreed to be contacted for potential enrollment in research studies (participants), 724 (15.3\%) declined future contact for research studies but provided anonymous screening data (anonymous participants), and $1762(37.1 \%)$ refused to participate under any circumstances. Thus, 2983 women completed the screening form, giving an overall agreement rate of $62.9 \%$. Rates of agreement varied over the course of the present study (Table 1), potentially owing to differences in the length of the various versions of the registry form. Versions 2 and 3 included an option to receive an information packet about perinatal depression. However, of the 2535 women who completed these two versions, only 87 (3.4\%) requested the material. 
Table 1

Numbers of women who agreed to participate by registry version. ${ }^{\mathrm{a}}$

\begin{tabular}{|c|c|c|c|c|}
\hline $\begin{array}{l}\text { Registry } \\
\text { version }\end{array}$ & $\begin{array}{l}\text { Agreed to } \\
\text { participate }^{\mathrm{b}}\end{array}$ & $\begin{array}{l}\text { Agreed to participate } \\
\text { anonymously }\end{array}$ & $\begin{array}{l}\text { Refused to } \\
\text { participate }^{\text {d }}\end{array}$ & Total \\
\hline 1 & $576(45.2)$ & $312(24.5)$ & $385(30.2)$ & 1273 \\
\hline 2 & $498(39.5)$ & $317(25.1)$ & $447(35.4)$ & 1262 \\
\hline 3 & $1210(54.8)$ & $191(8.6)$ & 809 (36.6) & 2210 \\
\hline \multicolumn{5}{|c|}{ a Values given as number (percentage) or number. } \\
\hline \multicolumn{5}{|c|}{ Gave permission for both retention of screening data and contact for research purposes. } \\
\hline \multicolumn{5}{|c|}{ c Gave permission for retention of anonymous screening data only. } \\
\hline \multicolumn{5}{|c|}{ d Denied permission for both retention of screening data and contact for research } \\
\hline
\end{tabular}

Between September 8, 2008, and June 9, 2011, 10 studies (including six intervention trials) used the registry to enroll a total of 630 participants (mean enrollment 230 women per year). A breakdown of the recruitment process for these studies is provided in Table 2.

Total EPDS scores were available for 2982 women, who had a mean score of $4.59 \pm 4.45$ (range 0.0-28.0). Among these women, 2488 (83.4\%) had an EPDS score below the recommended cutoff for minimal depression (<9), 247 (8.3\%) had scores within the at-risk range (9-11); and $247(8.3 \%)$ had scores suggestive of clinical depression $(>11)$. Individual EPDS item scores were available for 1986 (79.8\%) women. Excessive self-blame, excessive worry or anxiety, and feeling overwhelmed were the most frequently endorsed items (Table 3).

\section{Discussion}

The present study developed a screening and recruitment registry that was effectively implemented in seven clinics in the USA using limited resources. Overall rate of agreement to registry participation was high, as was rate of agreement to participate in further research, and the highest rate of agreement was obtained using a carefully crafted, concise screening and consent form combination. Furthermore, a high number of women were successfully enrolled into a range of research studies utilizing the registry as a recruitment tool.

Positive screening rates observed among the present study participants (using two cutoff EPDS scores) were consistent with expectations based on prevalence estimates of major and minor prenatal depression $[5,6]$. This finding highlights the importance of increased clinical and research attention to follow-up of depression screening results, such as monitoring of appropriate treatment use and outcomes. Few women requested the psychoeducational materials offered by research staff. Reasons for declining this material were not solicited, but it is possible that the women felt that they had received sufficient information from their doctors and/or did not feel the information would apply to them at that time.

The overall agreement rate recorded in the present study (62.9\%) was in line with rates reported from other studies in primary care
Table 3

Endorsement of individual Edinburgh Postnatal Depression Scale items among 1986 women.

\begin{tabular}{ll}
\hline Item & No. (\%) \\
\hline Not able to laugh or see the funny side of things & $397(20.0)$ \\
Not looking forward to things & $286(14.4)$ \\
Excessive self-blame & $1196(60.2)$ \\
Excessive worry or anxiety & $1190(59.9)$ \\
Panic and fear & $783(39.4)$ \\
Feeling overwhelmed & $1166(58.7)$ \\
Difficulty sleeping owing to mood disturbance & $520(26.2)$ \\
Feeling sad or miserable & $811(40.8)$ \\
Crying because of sad mood & $710(35.8)$ \\
"Hardly ever" or "sometimes" having thoughts of self-harm & $80(4.0)$ \\
"Often" having thoughts of self-harm & 0 \\
\hline
\end{tabular}

settings (53\%-87\%) [18-20]. However, the rates reported in the previous investigations were driven by recruitment to participate in one specific study rather than several different, unspecified trials as in the present study. The present strategy of developing a screening and consent registry to facilitate participation in multiple studies has the potential to reduce burden on patients, clinic staff, and researchers alike. Researchers specializing in perinatal mental health who aim to address the costly and burdensome outcomes of depression in the context of obstetrics care might, therefore, benefit from the adoption of an efficient screening system that allows for streamlined research recruitment into several related studies simultaneously.

One of the most important lessons learned regarding implementation of the registry was the need to develop strong and mutually beneficial relationships with obstetrics clinic staff. Several components were found to be crucial to meeting this objective. First, there was a need for discussion and communication with all stakeholders to identify mutual goals. Stakeholders can include, but are not limited to, department chairs, medical directors, clinic staff (physicians, nurses, medical assistants, office managers, and other staff), and members of the institutional review board. Second, a menu of options was offered by the researchers to assist with clinic needs. Such options might include in-service training, assistance with the mental health referral process, and involvement of trainees in research. Third, flexible tailoring of screening and recruitment procedures to different clinics while retaining key elements of standardization helped to accommodate the culture and work flow of each participating clinic. Finally, regular communication to maintain an ongoing presence with clinic staff, including in-person visits, was found to be essential, especially during times of clinic staff turnover.

Another key lesson of the present study involved facilitation of research participation rates. Consent and screening forms underwent several modifications to reduce burden on potential participants and to increase agreement rates, while ensuring a thorough consent process. A concise consent form was found to be essential to decreasing participant burden.

Table 2

Breakdown of the recruitment process among 10 studies that used the perinatal depression registry. ${ }^{\mathrm{a}}$

\begin{tabular}{|c|c|c|c|c|c|}
\hline Study & Enrolled & $\begin{array}{l}\text { Eligible but did not give } \\
\text { permission to be } \\
\text { contacted by research staff }\end{array}$ & $\begin{array}{l}\text { Eligible but could not } \\
\text { be contacted by } \\
\text { research staff }\end{array}$ & $\begin{array}{l}\text { Not interested in participating } \\
\text { after being contacted by } \\
\text { research staff }\end{array}$ & $\begin{array}{l}\text { Deemed ineligible to } \\
\text { participate after being } \\
\text { contacted by research staff }\end{array}$ \\
\hline 1 (O’Mahen et al. [28]) $^{\mathrm{b}}$ & 12 & 37 & 7 & 8 & 5 \\
\hline 2 (Marcus et al. [25]) ${ }^{\mathrm{b}}$ & 6 & 60 & 9 & 5 & 13 \\
\hline 3 (Mozurkewich et al. [29]) & 70 & 260 & 50 & 92 & 147 \\
\hline $4^{\mathrm{b}}$ & 13 & 20 & 7 & 4 & 2 \\
\hline 5 (Swanson et al. [30]) $^{\mathrm{b}}$ & 52 & 65 & 15 & 8 & 31 \\
\hline 6 (Muzik et al. [31]) & 69 & 31 & 23 & 16 & 493 \\
\hline 7 (Muzik et al. [32]) $^{\mathrm{b}}$ & 1 & 13 & 3 & 7 & 0 \\
\hline 8 & 211 & 150 & 1 & 1 & 1 \\
\hline 9 & 75 & 80 & 99 & 10 & 7 \\
\hline 10 & 121 & 61 & 4 & 29 & 224 \\
\hline
\end{tabular}

\footnotetext{
a Values are given as number.
}

b Intervention trial or included a treatment component. 
There are several strengths of the present study. First, the design incorporated a research recruitment tool into regular clinic operations to both improve research agreement rates and help to connect patients with clinical services through participation in trials. Second, the registry laid out a blueprint for a centralized recruitment core that other US-based sites could adapt or replicate. Third, the screening and recruitment strategy was found to be acceptable to both clinic staff and many of the participants. Finally, implementation of the registry facilitated ongoing collaboration among clinicians and researchers from a variety of disciplines. Such collaboration-coupled with the ability to reach and recruit large numbers of participants across multiple sites-is likely to be crucial to the success of future studies that aim to tackle the complex and multifaceted problem of untreated perinatal depression and its impact on public health.

Several limitations to the present study should also be considered. First, the registry represented a process implemented in one hospital system in the USA. Second, although a considerable age range was recorded within the registry population, the mean age of the participants (30.3 years) was not representative of the typical obstetrics population in the USA. Finally, the present study lacked systematic data regarding time or other resources saved as compared with other recruitment methods. A systematic implementation evaluation, including cost estimates of resources, would add valuable information. Other centers that develop such a screening program might anticipate using such a program for more extensive data collection and formulate the screen accordingly; however, it should be emphasized that short screens tend to generate high rates of return.

In conclusion, the screening and recruitment registry developed in the present study has the potential to improve detection of depression symptoms, increase research recruitment efficiency, reduce time and patient burden by combining these efforts, and provide a system for clinicians and researchers to work together. Future studies should aim to replicate the method at a national level, including collection of common measures and protocols across health centers to facilitate multicenter data collection.

Supplementary data to this article can be found online at http://dx. doi.org/10.1016/j.ijgo.2014.09.015.

\section{Conflict of interest}

The authors have no conflicts of interest.

\section{References}

[1] World Health Organization. The global burden of disease: 2004 update. http://www. who.int/healthinfo/global_burden_disease/GBD_report_2004update_full.pdf. Published 2008. Accessed July 31, 2014.

[2] Stewart WF, Ricci JA, Chee E, Hahn SR, Morganstein D. Cost of lost productive work time among US workers with depression. JAMA 2003;289(23):3135-44.

[3] Burke KC, Burke Jr JD, Rae DS, Regier DA. Comparing age at onset of major depression and other psychiatric disorders by birth cohorts in five US community populations. Arch Gen Psychiatry 1991;48(9):789-95.

[4] Spinelli MG. Antepartum and postpartum depression. J Gend Specif Med 1998;1(2): 33-6.

[5] Goodman JH, Tyer-Viola L. Detection, treatment, and referral of perinatal depression and anxiety by obstetrical providers. J Womens Health (Larchmt) 2010;19(3): 477-90.

[6] Gavin NI, Gaynes BN, Lohr KN, Meltzer-Brody S, Gartlehner G, Swinson T. Perinatal depression: a systematic review of prevalence and incidence. Obstet Gynecol 2005;106(5 Pt 1):1071-83.
7] Grote NK, Bridge JA, Gavin AR, Melville JL, Iyengar S, Katon WJ. A meta-analysis of depression during pregnancy and the risk of preterm birth, low birth weight, and intrauterine growth restriction. Arch Gen Psychiatry 2010;67(10):1012-24.

[8] Davis EP, Glynn LM, Schetter CD, Hobel C, Chicz-Demet A, Sandman CA. Prenatal exposure to maternal depression and cortisol influences infant temperament. J Am Acad Child Adolesc Psychiatry 2007;46(6):737-46.

[9] Marcus SM, Flynn HA, Blow FC, Barry KL. Depressive symptoms among pregnant women screened in obstetrics settings. J Womens Health (Larchmt) 2003;12(4): 373-80.

[10] Cox JL, Holden JM, Sagovsky R. Detection of postnatal depression. Development of the 10-item Edinburgh Postnatal Depression Scale. Br J Psychiatry 1987:150:782-6.

[11] Wisner KL, Chambers C, Sit DK. Postpartum depression: a major public health problem. JAMA 2006;296(21):2616-8.

[12] American College of Obstetricians and Gynecologists. Committee on Obstetric Practice. Committee opinion no. 453: Screening for depression during and after pregnancy. Obstet Gynecol 2010;115(2 Pt 1):394-5.

[13] Chadha-Hooks PL, Hui Park J, Hilty DM, Seritan AL. Postpartum depression: an original survey of screening practices within a healthcare system. J Psychosom Obstet Gynaecol 2010;31(3):199-205.

[14] Dietrich AJ, Williams Jr JW, Ciotti MC, Schulkin J, Stotland N, Rost K, et al. Depression care attitudes and practices of newer obstetrician-gynecologists: a national survey. Am J Obstet Gynecol 2003;189(1):267-73.

[15] Gul RB, Ali PA. Clinical trials: the challenge of recruitment and retention of participants. J Clin Nurs 2010;19(1-2):227-33.

[16] Grote NK, Spieker SJ, Lohr MJ, Geibel SL, Swartz HA, Frank E, et al. Impact of childhood trauma on the outcomes of a perinatal depression trial. Depress Anxiety 2012;29(7):563-73.

[17] Nanzer N, Sancho Rossignol A, Righetti-Veltema M, Knauer D, Manzano J, Palacio Espasa F. Effects of a brief psychoanalytic intervention for perinatal depression. Arch Womens Ment Health 2012;15(4):259-68.

[18] Gotlib IH, Whiffen VE, Mount JH, Milne K, Cordy NI. Prevalence rates and demographic characteristics associated with depression in pregnancy and the postpartum. J Consult Clin Psychol 1989;57(2):269-74.

[19] Yonkers KA, Ramin SM, Rush AJ, Navarrete CA, Carmody T, March D, et al. Onset and persistence of postpartum depression in an inner-city maternal health clinic system. Am J Psychiatry 2001;158(11):1856-63.

[20] Scholle SH, Haskett RF, Hanusa BH, Pincus HA, Kupfer DJ. Addressing depression in obstetrics/gynecology practice. Gen Hosp Psychiatry 2003;25(2):83-90.

[21] Chaudron LH, Szilagyi PG, Kitzman HJ, Wadkins HI, Conwell Y. Detection of postpartum depressive symptoms by screening at well-child visits. Pediatrics 2004;113(3 Pt 1): 551-8.

[22] Honey KL, Bennett P, Morgan M. Predicting postnatal depression. J Affect Disord 2003:76(1-3):201-10.

[23] Andersen JS, Olivarius Nde F, Krasnik A. The Danish National Health Service Register. Scand J Public Health 2011;39(7 Suppl.):34-7.

[24] Coleman MP, Forman D, Bryant H, Butler J, Rachet B, Maringe C, et al. Cancer survival in Australia, Canada, Denmark, Norway, Sweden, and the UK, 1995-2007 (the International Cancer Benchmarking Partnership): an analysis of population-based cancer registry data. Lancet 2011;377(9760):127-38.

[25] Marcus S, Lopez JF, McDonough S, MacKenzie MJ, Flynn H, Neal Jr CR, et al. Depressive symptoms during pregnancy: impact on neuroendocrine and neonatal outcomes. Infant Behav Dev 2011;34:26-34.

[26] Robins LN, Helzer JE, Croughan J, Ratcliff KS. National Institute of Mental Health Diagnostic Interview Schedule. Its history, characteristics, and validity. Arch Gen Psychiatry 1981;38(4):381-9.

[27] Rost K, Burnam MA, Smith GR. Development of screeners for depressive disorders and substance disorder history. Med Care 1993:31(3):189-200.

[28] O'Mahen H, Himle JA, Fedock G, Henshaw E, Flynn H. A pilot randomized controlled trial of cognitive behavioral therapy for perinatal depression adapted for women with low incomes. Depress Anxiety 2013;30(7):679-87.

[29] Mozurkewich EL, Clinton CM, Chilimigras JL, Hamilton SE, Allbaugh LJ, Berman DR, et al. The Mothers, Omega-3, and Mental Health Study: a double-blind, randomized controlled trial. Am J Obstet Gynecol 2013;208(4):313.e1-9.

[30] Swanson LM, Flynn H, Adams-Mundy JD, Armitage R, Arnedt JT. An open pilot of cognitive-behavioral therapy for insomnia in women with postpartum depression. Behav Sleep Med 2013;11(4):297-307.

[31] Muzik M, Bocknek EL, Broderick A, Richardson P, Rosenblum KL, Thelen K, et al. Mother-infant bonding impairment across the first 6 months postpartum: the primacy of psychopathlogy in women with childhood abuse and neglect histories. Arch Women Ment Health 2013;16(1):29-38.

[32] Muzik M, Hamilton SE, Rosenblum KL, Waxler E, Hadi Z. Mindfulness yoga during pregnancy for psychiatrically at-risk women: preliminary results from a pilot feasibility study. Complement Ther Clin Pract 2012;18(4):235-40. 